\title{
Financial Toxicity Following Post-Mastectomy Reconstruction: Consideration for a Novel Outcome Measure
}

\author{
Evan Matros, MD, MMSc ${ }^{1}$, and Anaeze C. Offodile II, MD, MPH ${ }^{2,3,4}$ \\ ${ }^{1}$ Section of Plastic and Reconstructive Surgery, Memorial Sloan Kettering Cancer Center, New York, NY; ${ }^{2}$ Department of \\ Plastic and Reconstructive Surgery, University of Texas MD Anderson Cancer Center, Houston, TX; ${ }^{3}$ Department of \\ Health Services Research, University of Texas MD Anderson Cancer Center, Houston, TX; ${ }^{4}$ Baker Institute for Public \\ Policy, Rice University, Houston, TX
}

Financial toxicity (FT) describes the multidimensional and downstream impact of the costs of care on the lives of patients and their families. Broadly, it can be categorized into three domains, namely material conditions (e.g. bankruptcy, high out-of-pocket [OOP] expenses), psychological response (e.g. increased worry, anxiety, poor quality-of-life), and coping behaviors (e.g. missed clinics, skipped medications). ${ }^{1}$ For a variety of reasons, cancer patients are particularly vulnerable to FT on account of the multimodal nature of therapies (e.g. chemotherapy, surgery, and radiation), high treatment intensity (especially at the end of life), long treatment time horizons, and the cumulative economic effects of managing disease recurrence or secondary cancers. FT has been associated with poor quality of life, treatment non-adherence, worse symptom burden, and decreased overall survival in cancer patients. $^{2-4}$

Subsequently, it has gained considerable attention in recent years as health care systems' have renewed their focus on patient-centeredness, following the influential Institute of Medicine Report: Crossing the Quality Chasm. ${ }^{5}$ Furthermore, several contemporaneous changes in the organization and financing of US healthcare have likely exacerbated the financial impact of treatment on patients' lives. As the US continues to outpace the rest of the world

(C) Society of Surgical Oncology 2021

First Received: 3 August 2021

Accepted: 31 August 2021;

Published Online: 15 September 2021

A. C. Offodile II, MD, MPH

e-mail: acoffodile@mdanderson.org in healthcare spending as a proportion of gross domestic product, many of these costs are passed on to patients in the private insurance market as well as Medicare recipients, with the latter having no spending limits for beneficiaries. ${ }^{6}$ One of the more recent relevant changes has been the proliferation of high-deductible health care plans. ${ }^{7}$ Although intended to limit indiscriminate spending by health care consumers, paradoxical effects have been measured, including delays in screening, later start time to chemotherapy, as well as missed care entirely. ${ }^{8-10}$ These findings are not only unique to the US health care system but have also been demonstrated in public health care systems such as the National Health Service in Italy. The conversation about FT extends to all aspects of the cancer care continuum, including breast reconstruction.

The current study entitled, 'Financial Toxicity in Breast Reconstruction: A National Survey of Women Who Have Undergone Breast Reconstruction After Mastectomy', builds upon preliminary works in this area. ${ }^{1-13}$ Using a 33-item survey administered to the Love Research Army, the authors aimed to evaluate whether patients experience FT attributable to breast reconstruction and to identify predictors. There were 922 respondents who were at least 1 year following postmastectomy breast reconstruction. Nearly one-quarter of women (23.8\%) reported FT from undergoing breast reconstruction, a finding that was associated with greater OOP expenses as well as major complications. Women who experienced self-reported FT were also less satisfied with information received from their care team regarding OOP expenses and would potentially decide against pursuing breast reconstruction again. Some limitations of the study help contextualize the findings further. The survey respondents may not be 
representative of the greater US population who undergo breast reconstruction as the overwhelming majority $(>90 \%)$ were Caucasian and privately insured; this may not be illustrative of those most vulnerable to financial distress. For simplicity, the authors defined FT dichotomously; however, it likely impacts patients along a continuum. We also cannot parse which aspects of care are directly attributed to breast reconstruction as opposed to the other aspects of cancer treatment (i.e. receipt of radiation or chemotherapy, ablative surgery). Finally, no information is provided about the type of health care plan, high versus low deductible, or if women had met the annual deductible at the time of reconstruction.

In framing the present results within the broader literature on FT, certain salient points warrant mention. First, breast reconstruction is preference-sensitive in several dimensions, such as indication (yes or no), timing (immediate or delayed), and particular subtype to pursue (prosthetic, autologous, and oncoplastic). As a result, each of these 'decision nodes' engender an opportunity for more robust shared decision making (SDM). SDM is a process that rests on a pre-existing patient-surgeon relationship and clear communication to delineate a patient's preferences, wants, and values with respect to a treatment course, risk/ benefits, and available alternatives. ${ }^{14}$ The study result that women who experienced FT were significantly more likely to decide against breast reconstruction underscores the need to embed cost transparency and discussions around the potential risks for FT based on established patient-level risk factors (e.g. income level, credit score, marital status) in the SDM process. ${ }^{11}$ Cost conversations could easily begin, with plastic surgeons engaging patients in discussion about whether or not they have a high versus low deductible health care plan as this will likely impact patient OOP expenses. Second, it is also concordant with the finding by Greenup et al. that among women with an income $<\$ 45,000$, treatment cost considerations were ranked as more important than breast reconstruction or preservation. ${ }^{15}$ Aligning treatment choices with a patient's preference hierarchy (e.g. OOP costs, recuperation downtime, breast shape or scar appearance) undergirds patientcentered, value-based breast reconstruction. The Institute of Medicine has strongly advocated for cost conversations in patient care encounters. ${ }^{16}$

Reconstructive surgeons have a vested interest in reducing the incidence and severity of FT, to the extent possible, because it dampens the psychosocial benefits of breast reconstruction and patient satisfaction. ${ }^{4}$ Of note, the authors also report that the various breast reconstruction subtypes, each with dissimilar cost structures, were not associated with differential risk profiles for FT, a finding reproduced in a recent single-institution study. ${ }^{12}$ Undoubtedly, addressing FT will require multi-level policy solutions that are beyond the scope of the present editorial and range from improving financial literacy (patient-level), to embedding financial navigators within breast cancer clinics (institution-level), to de-escalating the provision of low-value services (surgeon-level). ${ }^{11}$ Highlighting the high prevalence $(23.8 \%)$ and considerable sequelae of FT in breast reconstruction (i.e. decision regret, worse quality-oflife) is a crucial component of any meaningful effort. ${ }^{4,13}$ Future prospective, multicenter studies are needed to create (1) more generalizable results, (2) insights into the trajectory and ameliorating factors for FT, and (3) decompose the relative contributions of ablative surgery, chemotherapy, and breast reconstruction to FT risk. In conclusion, the authors should be congratulated for advancing our knowledge on this topic and for encouraging providers to engage in cost conversations with an eye towards enhanced SDM.

DISCLOSURE Anaeze Offodile II has received prior grant funding from Blue Cross Blue Shield and National Academy of Medicine unrelated to the current work. He has also received honorarium from the Department of Surgery at Indiana University and is an unpaid board member of the Patient Advocate Foundation. Evan Matros has no disclosures to declare.

\section{REFERENCES}

1. Altice CK, Banegas MP, Tucker-Seeley RD, Yabroff KR. Financial hardships experienced by cancer survivors: A systematic review. J Natl Cancer Inst. 2016;109(2):djw205. https://doi. org/10.1093/jnci/djw205.

2. Lentz R, Benson A, Kircher S. Financial toxicity in cancer care: Prevalence, causes, consequences, and reduction strategies. $J$ Surg Oncol. 2019;120(1):85-92.

3. Gordon LG, Merollini KMD, Lowe A, Chan RJ. A Systematic review of financial toxicity among cancer survivors: We can't Pay The Co-Pay. Patient. 2017;10(3):295-309.

4. Coroneos CJ, Lin YL, Sidey-Gibbons C, et al. Correlation between financial toxicity, quality of life, and patient satisfaction in an insured population of breast cancer surgical patients: a single-institution retrospective study. $J$ Am Coll Surg. 2021;232(3):253-63.

5. Committee on Quality Health Care in America, Institute of Medicine. Crossing the Quality Chasm: A New Health System for the 21st Century. Washington, DC: National Academies Press; 2001

6. Narang AK, Nicholas LH. Out-of-pocket spending and financial burden among medicare beneficiaries with cancer. JAMA Oncol. 2017;3(6):757-65.

7. Miller GE, Vistnes JP, Rohde F, Keenan PS. High-deductible health plan enrollment increased from 2006 to 2016, employerfunded accounts grew in largest firms. Health Aff. 2018;37(8):1231-7.

8. Wharam JF, Zhang F, Lu CY, et al. Breast cancer diagnosis and treatment after high-deductible insurance enrollment. J Clin Oncol. 2018;36(11):1121-7.

9. Wharam JF, Zhang F, Landon BE, Le Cates R, Soumerai S, RossDegnan D. Colorectal cancer screening in a nationwide highdeductible health plan before and after the affordable care act. Med Care. 2016;54(5):466-73.

10. Wharam JF, Zhang F, Wallace J, et al. Vulnerable and less vulnerable women in high-deductible health plans experienced 
delayed breast cancer care. Health Aff. 2019;38(3):408-15. http s://doi.org/10.1377/hlthaff.2018.05026.

11. Asaad M, Bailey C, Boukovalas S, et al. Self-reported risk factors for financial distress and attitudes regarding cost discussions in cancer care: A single-institution cross-sectional pilot study of breast reconstruction recipients. Plast Reconstr Surg. 2021;147(4):587e-95e.

12. Bailey C, Asaad M, Boukovalas S, et al. Understanding the relationship between breast reconstruction subtype and risk of financial Toxicity: A single-institution pilot study. Plast Reconstr Surgery. 2021;148(1):1e-11e.

13. Ganesh Kumar N, Berlin N, Hawley S, Jagsi R, Momoh A. Financial Toxicity in breast reconstruction: A national survey of women who have undergone breast reconstruction after mastectomy. Ann Surg Oncol. 2021. https://doi.org/10.1245/s10434021-10708-5.
14. Barry MJ, Edgman-Levitan S. Shared decision making-the pinnacle of patient-centered care. $N$ Engl $J$ Med. 2012;366(9):780-1.

15. Greenup RA, Rushing C, Fish L, et al. Financial costs and burden related to decisions for breast cancer surgery. J Oncol Pract. 2019;15(8):e666-676.

16. Levit L, Balogh E, Nass S. Delivering High-Quality Cancer Care: Charting a New Course for a System in Crisis. Washington, DC: National Academies Press; 2013.

Publisher's Note Springer Nature remains neutral with regard to jurisdictional claims in published maps and institutional affiliations. 